\title{
Répression des
}
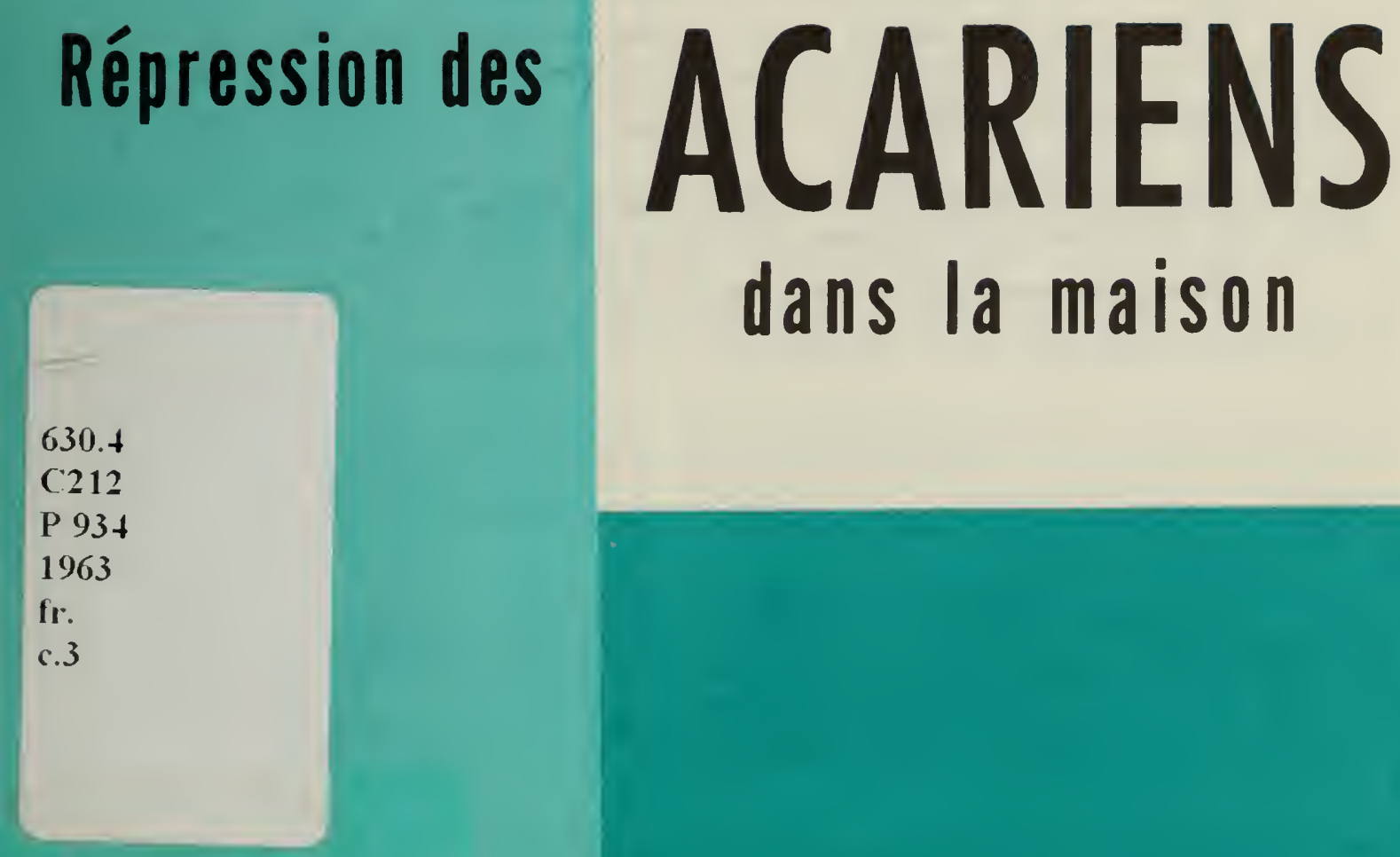

\section{dans la maison}

\section{MINISTEERE DE L'AGRICULTURE DU CANADA}




\section{TABLE DES MATIÈRES}

Utilis ation des acaricides

Répulsifs .......................................................... 4

Acariens des denrées alimentaires ......................... 4

Tétranyque du trèfle

Dermanys se des volailles ........................................ 8

Acarien des meubles …................................................ 9

Acarien de la paille ..................................................... 9

Tétranyques rouges des moisson s ......................... 10

Liponysse tropical du rat ........................................ 12

Dermanysse des souris …........................................... 12

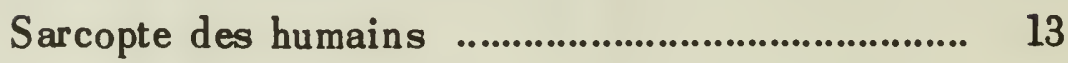

Choriopte de la gale de l'oreille ou otodecte ...... 14

Renseignements .......................................................... 14

ROGER DUHAMEL, M.S.R.C.

IMPRIMEUR DE LA REINE ET CONTRÔLEUR DE LA PAPETERIE OTTAWA, 1963

Cat. No: A43-934F 


\title{
Répression des
}

\author{
C. G. MacNay \\ Ferme expérimentale centrale, Ottawa
}

Les acariens sont des hôtes indésirables. Ils infestent les aliments, les meubles rembourrés, matelas, oreillers, particulièrement si la rembourrure est formée de fibre végétale.

Quelques acariens peuvent porter les germes de certaines maladies; d'autres, causer des dermatites et des réactions allergiques chez l'homme.

Les espèces les plus nombreuses: le tétranyque du trèfle et le dermanysse des oiseaux, s'introduisent dans les maisons, par les fentes et lézardes autour des fenêtres, des portes et des fondations.

La plupart des acariens sont si petits qu'ils sont à peine visibles à l'œil nu. Les nymphes et les adultes ont quatre paires de pattes, tandis que les larves n'en ont que trois. Chez plusieurs espèces, la femelle dépose des oufs; chez d'autres, les petits naissent vivants. Lorsque les conditions sont favorables, les acariens se multiplient avec une rapidité incroyable. Ils complètent leur cycle évolutif en deux ou trois semaines.

\section{UTILISATION DES ACARICIDES}

Les agents de destruction des acariens peuvent s'obtenir sous plusieurs formes. Insecticides et acaricides liquides se vendent sous forme de pulvérisations d'ambiance ou de contact, de pulvérisations rémanentes et d'aérosols. Les insecticides en poudre peuvent être employés tels quels ou diluées dans l'eau et appliqués en pulvérisations.

La plupart des insecticides domestiques sont également des acaricides efficaces.

Les acariens atteints par les pulvérisations de contact meurent rapidement, mais les résidus qui adhèrent aux surfaces traitées ont très peu d'effet.

Appliquer les pulvérisations d'ambiance ou de contact directement sur les acariens à l'aide d'un vaporisateur brouillard. La plupart des petits pulvérisateurs domestiques donnent de bons résultats. 
Les pulvérisations rémanentes ef la plupart des poudres agissent plus lentement, mais elles continuent pendant plusieurs jours à faire mourir les acariens. Utiliser un pulvérisateur à jet grossier afin de mouiller la surface traitée; si la surface est restreinte, se servir d'un pinceau. Traiter les surfaces fréquentées par les acariens, ainsi que les fentes et les lézardes où ils se cachent et par où ils pénètrent dans l'édifice.

Les poudres s'appliquent au moyen d'une boîte à couvercle perforé ou avec un sac en coton à fromage, mais il vaut encore mieux utiliser une poudreuse à main capable de bien faire pénétrer la poudre dans les fentes et les crevasses et atteindre aussi les endroits difficiles d'accès. II faut appliquer la poudre avec discrétion; tout excès est malpropre, peu économique et souvent inefficace.

Les aérosols servent surtout à abattre les insectes volants, mais ils peuvent détruire les acariens lorsqu'ils les atteignent directement.

Pour les applications d'acaricides à l'extérieur, on peut se servir d'un pulvérisateur à pression ou d'une poudreuse de jardin.

\section{Précautions}

Presque tous les acaricides sont toxiques pour l'homme. Suivre à la lettre les avertissements donnés sur le contenant.

Tenir les acaricides hors de la portée des enfants.

Manipuler avec soin et éviter de contaminer les aliments. Si un acaricide vient en contact avec la peau, se laver immédiatement à l'eau chaude et au savon.

\section{RÉPULSIFS}

On peut se protéger contre la plupart des acariens qui s'attaquent à l'homme: appliquer sur les vêtements et sur les parties exposées de la peau, un répulsif à base de diéthyltoluamide, phthalate de diméthyle, phthalate de dibutyle, ou benzoate de benzyle.

\section{ACARIENS DES DENRÉES ALIMENTAIRES}

Certains acariens infestent les aliments: farine, fromage, sucre, céréales, fruits secs, viandes, grains, confitures, gelées, graines et drogues. Ils se logent aussi dans les meubles rembourrés, matelas, oreillers. Ils sont très petits, incolores et couverts de longs poils spiniformes. Ils se reprodu isent rapidement et, en grand nombre, peuvent avoir une ode ur caractéristique. 
Tout dépot de poussière brunâtre dans les crevasses du fromage indique la présence des acariens. Cette poussière se compose d'acariens vivants ou morts, de dépouilles, d'excréments et de parcelles de fromage.

Lorsque ces acariens adhèrent à la peau, ils causent une irritation appelée "démangeaison de l'épicier"; $d$ 'autres espèces, vivant en association avec les acariens des grains, irritent aussi la peau. On peut soulager cette démangeaison en se lavant à l'eau froide ou tiède et en appliquant une lotion à la calamine ou au menthol. Absorbés avec les aliments, les acariens peuvent provoquer une irritation intestinale et la diarrhée.

\section{Répression}

Brûler les aliments infestés ou les étendre dans une lèchefrite et les chauffer dans le fourneau à une température de 130 à $140^{\circ} \mathrm{F}$. durant au moins une demi-heure. Bien surveiller la température lorsqu'il s'agit de la farine, des fruits secs et du fromage. Si les acariens adhèrent à certains aliments comme les fruits secs, les enlever avec un détergent et rincer les fruits. Sasser les céréales légèrement infestées.

A l'aide d'un aspirateur, nettoyer soigneusement les fentes et toutes les surfaces infestées. Vider les armoires. Pulvériser les tablettes, le fond des tiroirs, les fentes et crevasses avec un insecticide rémanent, tel que le malathion 3 p. 100 , lindane 0.5 p. 100, DDT 5 p. 100 ou chlordane 2 p. 100.

Dès qu'ils sont secs, couvrir de papier les tablettes et fonds des tiroirs. De cette façon les aliments ne viennent pas en contact avec les résidus de l'insecticide.

Les acariens peuvent continuer à apparaître, mais les résidus gardent leur efficacité pour quelques jours.

Sur les comptoirs ou autres surfaces où les aliments sont disposés directement, utiliser un insecticide ménager contenant:

de la roténone

un mélange de pyréthrines et de butoxyde de pipéronyle (Pyrénone)

tout autre synergiste dans une base de kérosène déodorisé.

Appliquer à l'aide d'un pinceau ou d'un pulvérisateur.

Il faut une concentration asse $z$ forte d'insecticide pour détruire les cirons du fromage.

On peut prévenir les infestation en prenant quelques précautions elémentaires.

- Garder les aliments au sec, autant que possible. 
- Conserver dans des contenants hermétiques les aliments susceptibles d'infestation.

- Nettoyer régulièrement tablettes et contenants.

- Nettoyer les contenaints chaque fois qu'on y remplace les aliments.

- Ne pas mélanger aliments frais et produits vieillis.

- Conserver le fromage au sec et à une température de 35 à $40^{\circ} \mathrm{F}$. Les acariens deviennent actifs et croissent à des températures supérieures à $40^{\circ} \mathrm{F}$.

\section{TÉTRANYQUE DU TRÈFLE}

Le tétranyque du trèfle s'introduit souvent dans les demeures. Il est de couleur brunâtre ou rougeâtre et c'est l'un des plus gros acariens qui s'attaquent aux plantes. Il envahit les murs extérieurs des maisons, surtout ceux qui sont exposés au soleil, et pénètre à l'intérieur par les fentes et les fissures des fenêtres, des portes et des fondations. Ces invasions se produisent ordinairement le printemps et l'automne; cependant dans les régions du sud elles ont aussi lieu durant l'hiver. Les ac ariens qui entrent dans la maison à l'automne, hivernent probablement au stade d'adulte, protégés qu'ils sont contre le froid. Ils peuvent aussi survivre à l'extérieur, au stade d'adulte, dans les régions de l'extrême sud de l'Ontario.

Cet acarien cause des dommages à une variété considérable de plantes de grande culture et de jardin, aux arbres fruitiers, aux arbres $d$ 'ornement et aux mauvaises herbes.

Il ne s'attaque pas aux humains et ne cause aucun dommage dans la maison, mais sa.présence peut être ennuyeuse et il laisse une tache rouge quand on l'écrase.

\section{Répression}

A l'intérieur-Utiliser l'un ou l'autre des acaricides rémanents suivants, préférablement à base d'huile.

$\begin{array}{lrlr}\text { malathion } & 3 \text { p. } 100 & \text { chlordane } & 2 \text { p. } 100 \\ \text { lindane } & 0.5 \text { p. } 100 & \text { Diazinon } & 0.5 \text { p. } 100 \\ \text { DDT } & 5 \text { p. } 100 & \text { dieldrine } & 0.5 \text { p. } 100\end{array}$

Appliquer sur les cadres des portes et fenêtres, le rebord des fenêtres et sur le plancher à l'entrée des portes, le long des plinthes, sans oublier les fentes et les crevasses. Il vaut mieux appliquer les pulvérisations sous forme de gouttelettes ou au moyen d'un pinceau en mouillant à fond les surfaces traitées. 
Lorsqu'il s'agit d'un acaricide sec, il faut le poudrer sur le rebord des fenêtres, de même que sur le plancher le long des plinthes et sous les portes. Se servir à cette fin d'un soufflet poudreur qui fasse pénétrer l'insecticide dans les fentes et crevasses par où les tétranyques pourraient $s$ 'introduire dans la maison.

Si l'on emploie un insecticide de contact contenant des pyréthrines ou de la roténone, on doit le pulvériser directement sur les acariens aussi souvent qu'il le faut pour arrêter l'invasion.

Les aérosols sont employés selon les instructions indiquées sur l'étiquette.

Vaporiser les meubles légèrement ou les essuyer avec un linge imbibé de kérosène raffiné. Appliquer un insecticide rémanent sur les châlits afin d'empêcher l'infestation des matelas. Laver et nettoyer à sec les fournitures de lit et les vêtements. Appliquer un insecticide de contact en pulvérisation légère sur les couvertures et les vêtements.

Utiliser fréquemment l'aspirateur et brûler les poussières ainsi recueillies.

A l'extérieur-tenir les alentours des fondations exempts de mauvaises herbes et de déchets et ne pas laisser l'herbe pousser jusqu'au mur.

Pulvériser l'herbe et les autres plantes sur une distance de 20 pieds des habitations, ainsi que la base des habitations sur une hauteur de trois pieds.

Utiliser l'un des insecticides suivants malathion émulsion 1 p. 100, ou 40 livres de poudre mouillable 25 p. 100 dans 100 gallons d'eau

Diazinon émulsion 1 p. 100 ou 40 livres de poudre mouillable 25 p. 100 dans 100 gallons d'eau chlorobenzilate émulsion 0.05 p. 100 ou 2 livres de poudre mouillable 25 p. 100 dans 100 gallons d'eau

Kelthane émulsion 0.05 p. 100

kérosène émulsion 10 p. 100

huile blanche émulsion 2 p. 100.

(huile d'été)

Appliquer environ 15 gallons de pulvérisation par 1,000 pieds carrés de sol. Éloigner enfants et animaux favoris des endroits pulvérisés jusqu'à ce que ce soit sec.

Les poudres contenant du soufre, du pyrèthre ou de la roténone sont aussi efficaces. Les insecticides à base d'huile, recommandés pour l'intérieur peuvent être pulvérisés sur les fondations, mais non sur les plantes. 
Calfeutrer les fissures autour des cadres de portes et de fenêtres de même que les lézardes dans les fondations. Accorder une attention particulière aux murs exposés au soleil.

\section{DERMANYSSES DES VOLAILLES}

Des nids d'oiseaux à l'extérieur, le dermanysse des volailles se transporte parfois à l'intérieur des habitations. Au cours de l'été, les nids peuvent devenir infestés. Les dermanysses s'attaquent périodiquement aux oisillons et aux adultes pour en sucer le sang. S'ils sont nombreux, ils peuvent pénétrer dans la maison généralement dès que les oiseaux ont quitté le nid.

Une fois à l'intérieur, les dermanysses envahissent les meubles, et parfois s'attaquent à l'homme. Ils causent des malaises considérables et provoquent quel quefois des éruptions qui ressemblent à une dermatite (voir remèdes contre les piqûres p. 11). Les invasions peuvent aussi provenir des poulaillers avoisinants ou de cages d'oiseaux infestés.

Les dermanysses peuvent survivre jusqu'à quatre ou cinq mois dans un poulailler après que toutes les volailles en ont été enlevées.

\section{Répression}

A l'intérieur-en général le traitement contre le tétranyque du trèfle (page 8 ) est aussi efficace contre les dermanysses des volailles.

Pour ce qui est des poulaillers, il faut nettoyer soigneusement les nids et la litière et pulvériser dans les fentes, les crevasses et autres cachettes surtout près des juchoirs et des nids avec un des mélanges suivants:

$\begin{array}{lrll}\text { malathion } & 3 \text { p. } 100 & \text { lindane } & 1 \text { p. } 100 \\ \text { Diazinon } & 0.5 \text { p. } 100 & \text { chlordane } & 2 \text { p. } 100 \\ \text { ronnel } & 1 \text { p. } 100 & \text { DDT } & 5 \text { p. } 100\end{array}$

Autres substances recommandées: mélange en parties égales d'un préservatif pour le bois à base d'huile anthracénique et de kérosène; solution à 4 p. 100 de sulfate de nicotine dans de l'eau; huile miscible à 3 p. 100 pour les arbres; kérosène; carbolineum; pétrole brut; huile à moteur additionnée d'une égale quantité de kérosène ou de créosote; lait de chaux contenant 5 p. 100 d'acide carbolique brut. Il ne faut pas employer de carbolineum, de créosote, ni de HCH dans les poulaillers de ponte, car ces produits peuvent communiquer un goût aux oeufs. Il n'est généralement pas nécessaire d'appliquer de traitement sur les volailles elles-mêmes, 
sauf dans le cas du liponysse: saupoudrer alors les oiseaux avec une poudre contenant 4 p. cent de malathion ou 2 à 5 p. 100 de lindane.

Si les cages deviennent infestées, on peut soit les ébouillanter, soit les badigeonner avec une solution de 20 gouttes d'iode dans une tasse d'eau chaude.

A l'extérieur-enlever tous les nids fixés aux bâtiments et traiter les surfaces infestées avec un des produits recommandés pour usage à l'intérieur.

\section{ACARIEN DES MEUBLES}

Les garde-manger, certains genres de meubles, et quelquefois le grain hébergent assez communément cet acarien blanc. Il n'endommage pas les textiles mais peut causer de la démangeaison. Lorsqu'ils sont nombreux ils deviennent une source d'ennuis graves.

L'acarien des meubles se nourrit de légumes secs et de matières animales et se plaît à l'humidité; c'est pourquoi on le trouve surtout dans les régions à climat très humide. Il infeste principalement les meubles rembourrés de fibre végétale. En cas de grave infestation, il peut envahir toute la maison.

\section{Répression}

Si les meubles sont infestés, enlever les bourrures de fibre végétale et les remplacer par d'autres matériaux, ou les faire fumiger à vide, au gaz cyanhydrique, par un spécialiste muni d'un permis à cette fin. Traiter les endroits infestés avec l'un des produits recommandés pour la répression des acariens des aliments (voir page 7). Tenir le degré d'humidité raisonnablement bas.

\section{ACARIEN DE LA PAILLE}

Cet acarien se nourrit ordinairement des larves de certains insectes des produits entreposés. De ce fait, il infeste communément le grain dans les minoteries, les entrepôts et les grains en transit. Dans les ports, il infeste aussi le copra, le coton en ballots, les sacs de blé ou d'orge, la paille ou le foin humide.

L'acarien de la paille passe par une phase presque invisible et c'est à ce stade qu'il s'attaque à l'homme. Il envahit souvent la peau et l'envers des vêtements des débardeurs et des travailleurs des mino- 
teries. Les gens qui couchent sur des matelas de paille ou de foin subissent quelquefois des piqûres douloureuses. A défaut de sa pâture normale, l'acarien de la paille s'attaque à n'importe quel animal. Il s'introduit presque toujours sous les vêtements avant de piquer et il s'attaque rarement à la peau découverte. Les piqûres sont suivies de graves démangeaisons, de rougeurs, de hausses de température, de maux de tête, de nausées et, en certains cas, de vomissements et de diarrhée bénigne. L'infection se développe souvent lorsqu'on gratte les piqûres. Les symptômes apparaissent quelquefois lorsque la peau vient en contact avec des acariens morts ou lorsque les acariens ou des poussières d'acariens sont aspirés, surtout si la personne est sujette aux allergies.

\section{Remèdes contre les piqûres}

Laver les piqûres avec de l'eau savonneuse fraiche ou tiède, puis appliquer: menthol, lotion à la calamine, poudre de talc, onguents analgésiques, ammoniaque, ou un mélange de 2 parties d'acide salicylique, 2 parties d'alcool et $I$ partie d'huile d'olive.

\section{Répression}

Brûler la rembourrure des matelas infestés et pulvériser sur l'endroit et l'envers du coutil. Les poudres contenant du soufre, 10 p. 100 de DDT, 5 p. 100 de chlordane ou 1 p. 100 de lindane sont également efficaces. Utiliser un répulsif à base de phthalate de diméthyle ou autre pour prévenir l'infestation du corps. Les onguents à base de soufre detruisent les acariens déjà présents sur le corps.

Détruire ou fumiger les restes de céréales infestées ou les faire chauffer à une température de $140^{\circ} \mathrm{F}$. pendant une heure.

Dans les cuisines infestées utiliser les pulvérisations recommandées pour la répression des acariens des aliments.

\section{TÉTRANYQUES ROUGES DES MOISSONS}

Les tétranyques rouges des moissons comprennent toutes les espèces de la famille des Trombiculidés; dans les Îles Britanniques, les acariens de cette famille sont connus sous le nom d'acariens des moissons. Les nymphes et les adultes de ce groupe ne sont pas très connus car ils ne s'attaquent ni aux humains ni aux animaux de la ferme. Toutefois, les larves s'attaquent à l'homme et aux animaux sauvages et domestiques. On les rencontre dans certaines régions du sud de l'Ontario et du Québec, 
surtout dans les maisons d'été. Ils apparaissent à la fin de l'été et à l'automne, dans les terrains incultes couverts $d$ 'herbages ou de mauvaises herbes.

Le parasite perce la peau, suce le sang et reste souvent en place jusqu'à ce qu'il soit repu. Les piqûres causent des irritations et des démangeaisons très ennuyeuses. Les tétranyques des moissons diffèrent des acariens de la paille en ce qu'ils piquent surtout les parties exposées du corps, mais ils peuvent aussi entrer sous les vêtements et à travers les tricots, les étoffes grossières. Le grattement des pi qûres peut donner lieu à de sérieuses infections de la peau.

\section{Remèdes contre les piqûres}

Retirer soigneusement les acariens de la peau et appliquer: alcool, iode, ammoniaque, camphre, chloroforme, ou vaseline phéniquée pour soulager l'irritation et prévenir l'infection.

\section{Rópression}

Nettoyer les régions infestées en coupant la pelouse et les mauvaises herbes. Tailler ou abattre les arbres afin de laisser pénétrer le soleil.

Avant d'entrer dans une région infestée, traiter les vêtements avec un répulsif contenant l'un des ingrédients suivants: diéthyltoluamide, benzyle, carbonate de diphényle, benzoate de phényle, phthalate de diméthyle, phthalate de dibutyle, ou benzoate de benzyle. La plupart des répulsifs contiennent un ou plusieurs de ces ingrédients. Ces produits protègent les vêtements, même après plusieurs lavages. Ils peuvent être appliqués en poudre sur le corps et les vêtements.

Pour éliminer ces acariens dans les régions incultes, appliquer des pulvérisations, des poudres de benzoate de benzyle ou de carbonate de diphényle à raison de cinq livres à l'acre. Les émulsions de malathion, chlordane, $\mathrm{HCH}$, toxaphène, et flavane sont efficaces dans les proportions de deux à quatre livres d'ingrédient actif par 20 à 25 gallons d'eau à l'acre.

Si les produits sont appliqués à l'état de poudre, et, à des dosages légèrement plus élevés, il y a moins de danger d'endommager les plantes. Utiliser le soufre à raison de 50 livres à l'acre. Ne pas utiliser de chlor dane, de $\mathrm{HCH}$ ou de toxaphène près des étangs ou des ruisseaux pe uplés de poissons.

Tenir enfants et animaux favoris éloignés des endroits pulvérisés, tant que l'herbe n'est pas sèche. 


\section{LIPONYSSE TROPICAL DU RAT}

Voilà un habitant des climats chauds, vu au Canada en de très rares occasions. Rien n'indique qu'il s'y soit établi. Grâce à sa petite taille, il passe souvent inaperçu, de sorte qu'on rejette ses méfaits sur les puces ou autres insectes. Dans les pays où il habite normalement, il s'attaque à l'homme, lorsque son hôte principal, le rat brun a été détruit. Sa morsure est suivie de démangeaison, de douleur et de petites tâches rouges ou de pustules aqueuses connues sous le nom de dermatite du liponysse du rat. Il s'attaque à n'importe quelle partie du corps, et si on se gratte, il peut en résulter une infection bactérienne. On rapporte qu'il peut transmettre aux humains le typhus endémique et la variole rickettsienne. (Voir remèdes contre les pi qûres, page 13).

\section{Répression}

Chasser les rats des édifices, dépotoirs et autres cachettes où cet acarien existe. Dans les endroits infestés, vaporiser les produits recommandés pour la répression du tétranyque du trèfle (voir page 8). Le pyrèthre et le léthane employés ensemble ou séparément dans du kérosène raffiné ont donné de bons résultats. Essuyer les meubles avec un chiffon trempé dans le kérosène.. Si l'infestation persiste, il faut faire fumiger l'édifice par un fumigateur de métier. On peut détruire les acariens sur les souris de laboratoire au moyen d'une poudre à 10 p. 100 d'ovex. Plonger les cages dans une forte solution désinfectante. Dans la chasse de cet acarien, examiner surtout les endroits chauds de la maison, par exemple, près des radiateurs, des tuyaux à air chaud et des bouches de chaleur.

\section{DERMANYSSE DES SOURIS}

Ce fléau des rats et des souris véhicule la variole rickettsienne chez les humains. Lorsqu'il n'est pas sur son hôte naturel, le dermanysse erre dans les habitations et les autres bâtiments, spécialement dans les endroits chauds.

\section{Répression}

Mêmes recommandations que pour le liponysse tropical du rat. 


\section{SARCOPTE DES HUMAINS}

C'est un parasite exclusif de l'homme, jamais des habitations. Cependant, les symptômes de l'infestation ressemblent à ceux causés par d'autres insectes ou acariens et peuvent fácilement prêter à confusion.

La femelle s'introduit sous l'épiderme, elle s'y nourrit et dépose ses oeufs dans la galerie ainsi pratiquée. Ordinairement, le mâle rampe à la surface de la peau. Le cycle évolutif varie de deux à six semaines.

Les épidémies de gale vont souvent de pair avec la malpropreté et l'encombrement. Le premier symptôme est une démangeaison qui se produit surtout la nuit. A mesure que l'infestation progresse, la gale apparait entre les doigts, aux poignets, aux aisselles, et à la partie charnue des cuisses. Le sarcopte n'attaque pas au-dessus du cou, bien qu'une autre espèce très rare se loge dans le cuir chevelu et les autres parties de la tête. Le visage de la victime devient pâle et hagard, faute de sommeil. Certaines personnes deviennent allergiques aux sécrétions du parasite.

\section{Répression}

La répression exige un traitement minutieux et la stérilisation des vêtements et de la literie du patient. Il est donc préférable de consulter un médecin.

Le benzoate de benzyle peut être utilisé à diverses concentrations. Appliquer deux fois par jour pendant cinq jours un mélange de 25 p. 100 de benzoate de benzyle dans une lotion à la calamine. L'armée américaine utilisé un concentré composé des ingrédients suivants, au poids:

$\begin{array}{lr}\text { benzoate de benzyle } & 68 \text { p. } 100 \\ \text { DDT } & 6 \text { p. } 100 \\ \text { benzocaîne } & 12 \text { p. } 100 \\ \text { Tween } 80 & 14 \text { p. } 100\end{array}$

Diluer à raison d'une partie dans cinq parties d'eau et appliquer avec une éponge ou un vaporisateur. Se laver à l'eau chaude et au savon avant l'application. Le lindane donne de bons résultats à la concentration de 0.05 p. 100 dans une émulsion à base d'eau, d'huile d'olive ou de crème à démaquiller.

Dans le traitement contre le sarcopte, il est important d'appliquer le remède du cou aux pieds; la moindre surface oubliée peut donner lieu à une réinfestation.

Il importe également de stériliser à l'e au bouillante tous les vêtements et la literie qui ont touché au patient avant et durant le traitement. 
Faire chauffer les lainages à une température de 130 à $140^{\circ} \mathrm{F}$. pendant au moins une demi-heure ou les plonger dans une solution de nettoyage tel un solvent volatile de pétrole.

\section{CHORIOPTE DE LA GALE DE L'OREILLE OU OTODECTE}

Chiens, chats, lapins et renards sont victimes du choriopte de la gale de l'oreille. Il irrite l'oreille, cause la surdité et le manque de coordination des mouvements.

Comme le sarcopte des humains, il reste sur l'animal et n'est pas réellement un acarien de l'habitation. Toutefois il est assez commun et passe inapercu car on ne le voit pas facilement.

Si les animaux manifestent des signes de l'irritation de l'oreille, il faut examiner les oreilles. La présence de croûtes sèches est généralement le résultat de l'oeuvre des chorioptes.

\section{Répression}

Nettoyer l'intérieur de l'oreille à l'aide d'un tampon de coton tenu avec des pinces et trempé dans l'une des solutions ci-après mentionnées. Éviter de blesser le tympan.

$\begin{array}{lc}\text { phénol (acide carbolique) } & 1 \text { p. } 100 \text { dans la glycérine } \\ \text { lindane } & 0.25 \text { p. } 100 \text { dans l'huile d'olive } \\ \text { DDT } & 5 \text { p. } 100 \text { en émulsion } \\ \text { soufre } & \text { en mélange crémeux } \\ & \text { dans l'huile d'olive }\end{array}$

Avec un tampon propre enlever soigneusement le pus et autres matières et, appliquer encore de la solution.

Répéter le traitement dans les sept jours, afin de détruire les chorioptes nés des oeufs présents au début du traitement. Afin de protéger les animaux contre une nouvelle infestation nettoyer les cases et locaux avec une solution chaude de coltar et de créosote.

\section{RENSEIGNEMENTS}

Pour obtenir de plus amples renseignements, consulter l'agronome local, l'entomologiste provincial ou s'adresser soit au laboratoire local d'entomologie du ministère de l'Agriculture du Canada, soit à la Section de l'information scientifique, Ferme expérimentale centrale, Ottawa. 
Dans la présente publication, on s'est servi de quelques noms commerciaux parce que leurs noms techniques ne sont pas connus du grand public et qu'il n'existe pas de noms communs officiels pour les ingrédients actifs.

\section{NOMS SCIENTIFIQUES DES ACARIENS}

Acarien des meubles

Acarien de la paille

Acariens des denrées alimentaires

Choriopte de la gale de l'oreille ou otodecte

Dermanysse des souris

Dermanysse des volailles

\section{Liponysse tropical du rat}

Sarcopte des humains

Tétranyque du trèfle
Glycyphagus domesticus (De Geer) Pyemotes véntricosus (Newport) Espèces de Acarus et de Tyrophagus Octodectes cynotis (Hering), Chorioptes bovis (Hering), et autres Allodermanyssus sanguineus (Hirst) Dermanyssus gallinae (De Geer) Ornithonyssus sylviarum (Canestrini \& Fanzago) et autres

Ornithonyssus bacoti (Hirst)

Sarcoptes scabiei (Linnaeus) Bryobia praetiosa Koch

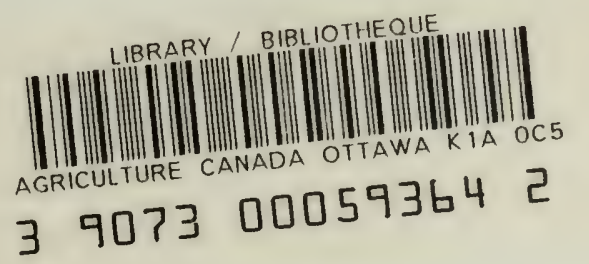


On peut se procurer d'autres exemplaires de cette publication en adressant sa demande à la :

DIVISION DE L'INFORMATION

MINISTÉRE DE L'AGRICULTURE DU CANADA Ottawa 